\title{
The Efficacy of Puromycin and Adriamycin for Induction of Glomerular Failure in Larval Zebrafish Validated by an Assay of Glomerular Permeability Dynamics
}

\author{
Sebastien Andrew Rider,' Finnius Austin Bruton,' Richard George Collins,2 \\ Bryan Ronald Conway, and John James Mullins ${ }^{1}$
}

\begin{abstract}
Defects in the glomerular filtration barrier (GFB) play a major role in the onset of human renal diseases. Highly ramified glomerular cells named podocytes are a critical component of the GFB. Injury to podocytes results in abnormal excretion of plasma proteins, which can lead to chronic kidney disease. The conserved paired nephron of larval zebrafish is an excellent model for assessing glomerular function and injury. The efficacy of two known podocyte toxins was tested to refine models of acute podocyte injury in larval zebrafish. The validated compound was then used to test a novel assay of the dynamics of abnormal protein excretion. Injected adriamycin was found to be unsuitable for induction of glomerular injury due to off-target cardiovascular toxicity. In contrast, puromycin treatment resulted in a loss of discriminative filtration, measured by excretion of $70 \mathrm{kDa}$ dextran, and podocyte effacement confirmed by electron microscopy. The dynamics of dextran excretion during puromycin injury modeled the onset of glomerular damage within 24 hours postinjection. These data validate puromycin for induction of acute podocyte injury in zebrafish larvae and describe a semihigh-throughput assay for quantifying the dynamics of abnormal protein excretion.
\end{abstract}

Keywords: puromycin, adriamycin, podocyte, glomerulus, pharmacological, kidney

\section{Introduction}

A PPROXIMATELY 1 MILLION glomeruli filter $90 \mathrm{~L}$ of blood in each human kidney every day. ${ }^{1}$ The precise filtration of blood constituents depends on the maintained integrity of the glomerular filtration barrier (GFB). The GFB comprises two highly specialized cell types separated by a type IV collagen and laminin-rich glomerular basement membrane $(\mathrm{GBM}) .^{2}$ The fenestrated endothelium forming the walls of glomerular capillaries is enwrapped by podocytes. Podocytes are highly ramified epithelial cells with primary and secondary foot processes, the structure of which depends on their actin cytoskeleton. ${ }^{3}$ The filtration slits between interdigitated podocyte foot processes comprise highly specialized gap junctions called slit diaphragms (SDs). The SD protein complex forms the barrier to protein filtration and is essential for maintained podocyte structure and function. ${ }^{4}$

All identified monogenic mutations associated with nephrotic syndrome are in podocyte-specific genes, revealing the essential role of these specialized cells for glomerular function. Disease-causing recessive or dominant autosomal mutations have been identified, among others, in nephrin (NPHS1), ${ }^{5}$ transient receptor potential channel 6 (TRPC6), ${ }^{6}$ Wilm's tumor suppressor gene 1 (WT1), ${ }^{7}$ and podocin (NPHS2). ${ }^{8}$ Most glomerular injuries are, however, not hereditary, but acquired in immune or nonimmune diseases, with $75 \%$ of cases having an unknown cause. ${ }^{9}$

Podocyte effacement, the gradual simplification of the interdigitating foot process pattern, is a key pathology common to most glomerular diseases. ${ }^{9,10}$ The breakdown of the GFB in disease, or as a result of drug toxicity, causes proteinuria, the abnormal filtration of plasma proteins, including albumin. Proteinuria associated with nephrotic syndrome results in edema, likely due to increased sodium retention by the distal nephron. ${ }^{11}$

Experimental animal models are required that recapitulate the molecular events that manifest during glomerular injury and the sequelae of pathological responses that lead to

\footnotetext{
${ }^{1}$ Univeristy/BHF Centre for Cardiovascular Science, The Queen's Medical Research Institute, Little France, The University of Edinburgh, Edinburgh, United Kingdom.

${ }^{2}$ Edinburgh College of Art, University of Edinburgh, Edinburgh, United Kingdom.

(C) Sebastien Andrew Rider et al. 2018; Published by Mary Ann Liebert, Inc. This Open Access article is distributed under the terms of the Creative Commons License (http://creativecommons.org/licenses/by/4.0), which permits unrestricted use, distribution, and reproduction in any medium, provided the original work is properly cited.
} 
glomerulosclerosis and the loss of functioning glomeruli. ${ }^{9}$ In rodents, acute nephrosis may be experimentally induced through insult to podocytes by nephrotoxic compounds. Injection of the antibiotic, puromycin aminonucleoside (PAN), causes either a mild or reversible glomerular damage resembling minimal change disease, or more severe lesions that mimic human focal segmental glomerulosclerosis (FSGS), ${ }^{12,13}$ the latter a hallmark of lasting and progressively damaging chronic kidney diseases. ${ }^{14}$ Similarly, a single injection of the chemotherapy drug, adriamycin, causes a severe form of FSGS in rats and mice. ${ }^{15}$

With its unique set of experimental advantages, including genetic tractability and unparalleled opportunity for in vivo experimentation, zebrafish have become a valuable animal model for renal research. The small size, rapid development, and translucency of larval zebrafish offer excellent opportunities for whole-animal chemical and genetic screening ${ }^{16}$ and, recently, for studies of renal cancer. ${ }^{17-21}$ Within the first 3 days of life, the pronephros of larval fish becomes functional and capable of discriminative filtration. ${ }^{22,23}$ Mature pronephric podocytes in larval zebrafish are anatomically similar to their mammalian counterparts and are nonmigratory, including during repair. ${ }^{24-26}$ With a developed glomerulus, segmented tubules, and renin-expressing cells, the single paired nephron of the zebrafish pronephros shares remarkable anatomical and functional similarity with the metanephric mammalian nephron. 22,23,27-33

Due to inherent limitations in current methods, the zebrafish model requires novel and refined renal injury techniqiues. ${ }^{34} \mathrm{~A}$ particular drawback to pharmacological models of acute renal failure in larval fish is that they are terminal, which limits the potential for follow-up studies. ${ }^{34}$ Genetic approaches for induction of glomerular injury have successfully utilized nitroreductase (NTR) ${ }^{35-37}$ to induce ablation of podocytes by cytotoxic metabolization of metronidazole (MTZ) ${ }^{38}$ This recapitulates proteinuria and has been successfully used to demonstrate the regenerative capacity of fish podocytes postinjury. ${ }^{25,35,37}$ However, the NTR/MTZ system is limited by side effects of MTZ and the prolonged time required for induction of apoptosis.

While also often limited by off-target effects, pharmacological approaches are quick to implement and may be applied to a variety of wild-type or transgenic lines. Current methods for the pharmacological disruption of podocytes in zebrafish utilizing puromycin result in an increased glomerular permeability. ${ }^{39}$ A comparison of pharmacological agents for induction of podocyte injury in zebrafish is required to identify compounds exhibiting the greatest potency with the lowest off-target effects.

The aims of the present study were twofold, (1) to compare puromycin and adriamycin for pharmacological induction of glomerular injury in zebrafish larvae with an established GFB and (2) to develop a high-throughput assay to measure the in vivo dynamics of abnormal protein excretion.

Adriamycin and puromycin were tested for their ability to induce structural podocyte damage and increased glomerular permeability. Increased permeability was determined by the excretion of normally retained dextran, and changes in podocyte morphology were assessed by transmission electron microscopy (TEM). As glomerular function is tightly associated with cardiovascular function in larval fish, ${ }^{40}$ the effects of these compounds on heart rate and blood flow were also tested.
After validating a puromycin model of glomerular injury, a novel dextran excretion assay was developed to assay the dynamics of glomerular permeability. Our study provides both a defined pharmacological model of acute podocyte injury and a novel assay enabling the measurement of protein excretion dynamics during glomerular injury and repair.

\section{Materials and Methods}

\section{Zebrafish and husbandry}

All experiments utilizing fish $>5 \mathrm{dpf}$ (days postfertilization) were approved by the local ethics committee and conducted in accordance with the Animals (Scientific Procedures) Act 1986 in a UK Home Office-approved establishment. Unless stated otherwise, wild-type WIK zebrafish (Danio rerio) were maintained at $28.5^{\circ} \mathrm{C}$ in $1 \times$ conditioned water $(\mathrm{CW})^{40}$ containing $0.1 \%(\mathrm{w} / \mathrm{v})$ methylene blue. Where required, fish were anesthetized in CW containing $40 \mu \mathrm{g} \mathrm{mL}^{-1}$ MS-222 (tricaine methanesulfonate). Forty micrograms per milliliter MS-222 is sufficient for anesthesia without significant effects on the heart rate. ${ }^{41}$ Microinjections and microscopy were carried out at room temperature $\left(26^{\circ} \mathrm{C} \pm 0.5^{\circ} \mathrm{C}\right)$.

\section{Microinjection of dextran and pharmacological agents}

Dextran and pharmacological agents were administered under anesthesia by intravenous microinjection through the cardiac sinus venosus (SV) that drains the common cardinal vein $(\mathrm{CCV}){ }^{42}$ All injections were administered in a $1-\mathrm{nL}$ volume. A successful injection was determined as a $100 \%$ delivery into the cardiovascular system. Injections where solutions ended up in the yolk, or in the pericardial sac, were deemed as unsuccessful; 5\% (w/v) $70 \mathrm{kDa}$ FITC-dextran was dissolved in phosphate-buffered saline (PBS). PAN (\#15509; Cayman Chemical Company) was administered at 5 or $25 \mathrm{mg}$ $\mathrm{mL}^{-1}(17.0-85.0 \mathrm{mM})$ and adriamycin (doxorubicin hydrochloride) (\#15007; Cayman Chemical Company) at 1,2, or $4 \mathrm{mg} \mathrm{mL}^{-1}(1.7,3.4,6.9 \mathrm{mM})$. Shams were administered with vehicle (PBS) only.

\section{Determination of the dose for induction of glomerular injury}

Three dpf fish were injected respective treatment compounds, or vehicle (PBS), and separated into Petri dishes in triplicate. Fish were examined immediately after injection and at 24 and 48 hpi (hours postinjection). At each time point, fish were scored for percentage survival and overt pericardial sac edema.

\section{Dextran clearance assay}

The clearance of $70 \mathrm{kDa}$ FITC-dextran was determined by measuring fluorescence intensity in the dorsal aorta.

In summary, postinjection, fish recovered in $\mathrm{CW}$ (without methylene blue) before brief anesthesia and mounting in 3\% methylcellulose for baseline imaging at $3 \mathrm{hpi}$. Sagittal images of FITC fluorescence in the dorsal aorta were taken using a stereomicroscope with fixed parameters. Embryos were returned to $\mathrm{CW}$ and standard conditions immediately after each imaging time point. For each fish, the mean intensity of three areas of the dorsal aorta was measured using Fiji-National Institutes of Health (NIH). Mean fluorescence intensity 
values were normalized against the baseline fluorescence and percentage intensity change was calculated.

\section{Heart rate and venous erythrocyte velocity}

After respective treatments, $3 \mathrm{dpf}$ embryos were left to recover in $\mathrm{CW}$ for three hours before mounting in 3\% methylcellulose and immediate imaging under anesthesia. Bright-field videos over $120 \mathrm{~s}$ in length were acquired of the dorsal aorta at 50 frames per second. Single erythrocytes were then tracked through the cardinal vein anterior of the cloaca. The distance traveled by three erythrocytes per embryo was measured using Fiji and venous erythrocyte velocity calculated in $\mu \mathrm{m} \mathrm{s}^{-1}$. Heart rate was assessed similarly by recording the video at a frame rate of 10 frames per second and heartbeats counted manually over a 10 -s period. Both videos were taken of a lateral orientation.

\section{Dextran excretion assay in a modified 96-well plate}

Postinjection, fish were left to recover for $1 \mathrm{~h}$ in $\mathrm{CW}$ (without methylene blue) before distribution into a modified black, flatbottomed, 96-well Microfluor $1^{\circledR}$ plate (Nunc). Two hundred fifty-micrometer slits were used to create 48 paired wells (Fig. 4A). In each well pair, one well contained a single fish, leaving the second well free for measurements of FITC intensity in the CW (Fig. 4B). The two well pairs were filled with $\sim 790 \mu \mathrm{L} \mathrm{CW}$ (without methylene blue) and covered with realtime polymerase chain reaction (qPCR) (Sarstedt) film, leaving no air gap between the water and film. FITC intensity was read once every $15 \mathrm{~min}$ for $48 \mathrm{~h}$ at a $\lambda_{\mathrm{ex}}$ of $485 \mathrm{~nm}$ and $\lambda_{\mathrm{em}}$ of $535 \mathrm{~nm}$. At the end of each assay, fish survival was confirmed.

\section{TEM and image analysis}

Three dpf fish were injected with $25 \mathrm{mg} \mathrm{mL}^{-1}$ puromycin or vehicle. At 8 and $24 \mathrm{hpi}$, fish were fixed for TEM as described by Lyons et al. ${ }^{43}$ Briefly, fish were fixed in modified Karnovsky's solution (2\% glutaraldehyde and $4 \%$ paraformaldehyde in $0.1 \mathrm{M}$ sodium cacodylate buffer), postfixed in $2 \%$ osmium tetroxide in $0.1 \mathrm{M}$ imidazole and $0.1 \mathrm{M}$ sodium cacodylate, stained en bloc in saturated uranyl acetate, and dehydrated into $100 \%$ acetone. All of these steps were accelerated using microwave stimulation with a Panasonic microwave with inverter technology. Temperature throughout was maintained at $15^{\circ} \mathrm{C}$ in a cooled water bath. Following dehydration, embryos were embedded in Epon. Ultrathin sections $(60 \mathrm{~nm}$ thick) were cut from selected areas and mounted on Formvar/carbon-coated copper slot grids. After drying, these were stained in $1 \%$ aqueous uranyl acetate and Reynold's lead citrate, and then viewed in a JEOL JEM-1400 Plus TEM.

Representative images were collected on a GATAN OneView camera for analysis of GFB structural changes using Fiji, as similarly performed by Benchimol de Souza et al. ${ }^{44}$ The mean podocyte frequency per $\mu \mathrm{m}$ length (of basement membrane) and mean podocyte width were determined in all clearly identifiable sections of the GFB (Fig. 3A).

\section{Statistical analysis}

All data were statistically analyzed and graphed with GraphPad Prism 7 (La Jolla, CA). All percentage data were arcsine transformed before analysis. Dextran clearance from the dorsal aorta, heart rate, and blood flow were subjected to two-way ANOVA. Foot process width and foot process number per $\mu \mathrm{m}$ GBM were analyzed by one-way ANOVA. Dextran excretion data were analyzed by linear regression and comparison of regression slopes. The first derivative of excretion data was determined for individual fish. Differences between the mean first derivative of control and treatment groups were determined for measures between 0-24 hpi and 24-48 hpi using $t$-tests. All means are reported with a standard error of the mean.

\section{Results}

\section{Determination of doses for induction of pharmacological injury}

The dose of a single intravenous injection of puromycin or adriamycin required to cause edema without effects on survival was tested. Achieving successful intravenous injections is considerably easier, and consequently more accurate, in $3 \mathrm{dpf}$ fish due to the large CCV and SV. Due to a narrowing of the CCV by $4 \mathrm{dpf}$, injections in older larval fish are much more challenging and error prone. Adriamycin affects angiogenesis in mammals ${ }^{45}$ and, similarly in fish, affects formation of the subintestinal vessels and eye development. ${ }^{46}$ The measurement of FITC in peripheral vessels is therefore potentially problematic. Similarly, FITC measurements in the heart can be obscured by accumulation of dextran in the pericardial sac, particularly in cases of edema. To minimize any potential confounding effects on FITC measurements and to allow normalization to baseline values, measurements of FITC were determined in the dorsal aorta, as similarly performed by Rider et al. $^{40}$

As podocytes exhibit elaborate primary processes and numerous interdigitating foot processes by $3 \mathrm{dpf},{ }^{26}$ this age was selected for the injection of compounds to induce pharmacological injury. Edema is readily observed as a result of acute renal failure in larval fish. ${ }^{21,39,40,47}$ The concentration of puromycin was partially based on previous studies, ${ }^{39}$ and following preliminary studies, three concentrations of adriamycin were tested. One hundred percent survival was observed at both tested doses of puromycin $\left(5\right.$ or $25 \mathrm{mg} \mathrm{mL}^{-1}$ ). Only the higher dose of puromycin resulted in edema (Fig. 1A), which was observed from $24 \mathrm{~h}$ in $45 \%$ of fish (Fig. 1B).

The higher doses of adriamycin $\left(2\right.$ and $\left.4 \mathrm{mg} \mathrm{mL}^{-1}\right)$ decreased survival to $90 \%$ after $24 \mathrm{~h}$. Two milligrams per milliliter adriamycin resulted in transient edema in $>10 \%$ of fish, conversely $4 \mathrm{mg} \mathrm{mL}^{-1}$ resulted in persistent edema in $75 \%$ of larvae, some of which had remaining yolk reserves suggestive of stunted development (Fig. 1A, C). Due to the mortality and gross morphological changes associated with the highest dose of adriamycin, only 1 and $2 \mathrm{mg} \mathrm{mL}^{-1}$ concentrations were taken forward for further study.

\section{Effects of puromycin and adriamycin on renal permeability and cardiovascular function}

Both puromycin ${ }^{12}$ and adriamycin ${ }^{15}$ are known to disrupt the GFB in mammals. While the effect of injected adriamycin on developed podocytes is untested in fish, puromycin has been shown to disrupt the fish GFB. ${ }^{39}$ Puromycin and adriamycin were compared as pharmacological podocyte injury models in larval fish by testing both target effects 

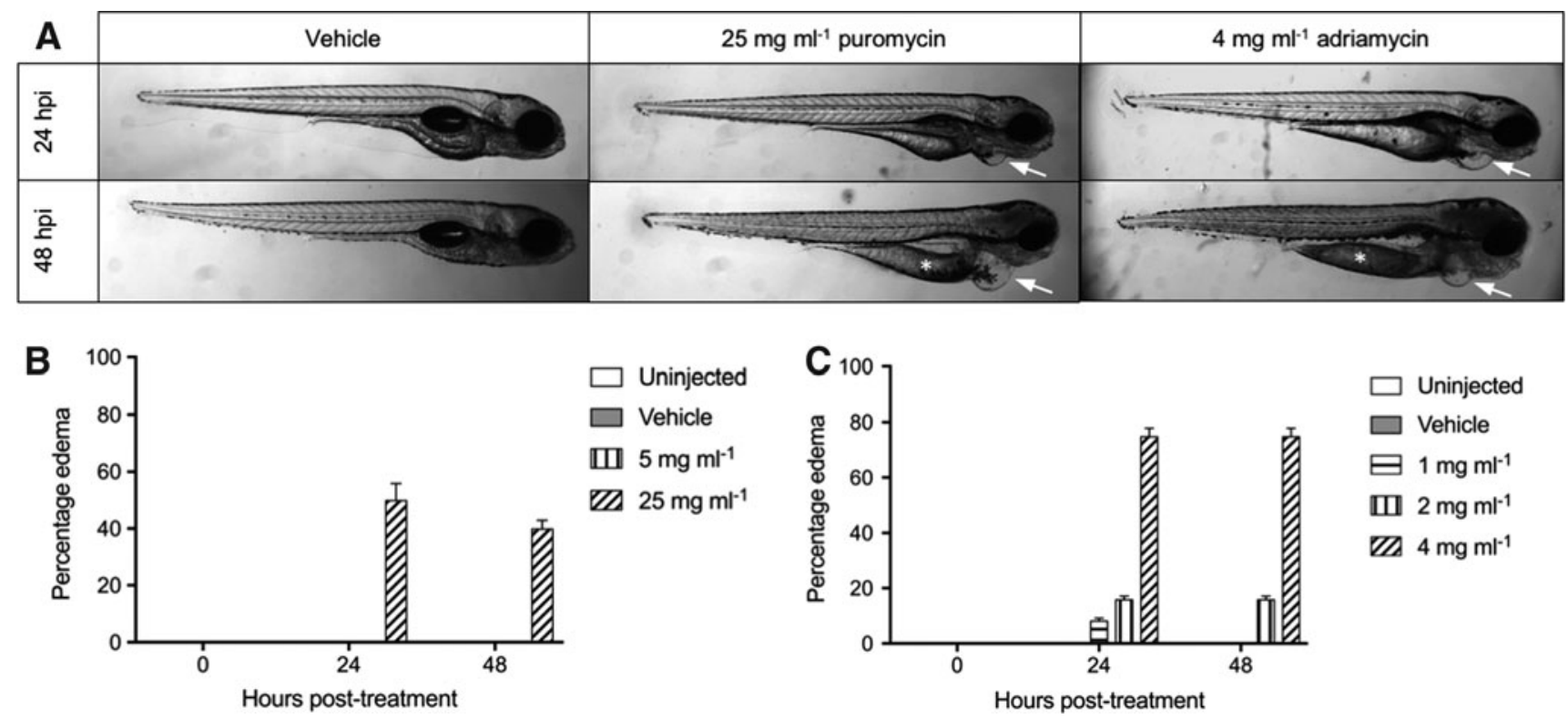

FIG. 1. Determination of pharmacological injury doses- $1 \mathrm{~nL}$ injection at $3 \mathrm{dpf}$. (A) Representative images showing pericardial edema (arrows) and remaining yolk reserves $\left(^{*}\right)$ presented in a portion of larvae administered adriamycin or puromycin. (B) Five milligrams per milliliter puromycin does not result in edema. Increasing the puromycin dose to $25 \mathrm{mg}$ $\mathrm{mL}^{-1}$ causes edema in $>40 \%$ of fish from $24 \mathrm{hpi}$ (mean \pm SEM). (C) Four milligrams per milliliter adriamycin causes edema in $>75 \%$ of larvae by $24 \mathrm{hpi}$ (mean \pm SEM). SEM, standard error of the mean; dpf, days postfertilization; hpi, hours postinjection.

on glomerular permeability and side effects on cardiovascular function.

Only the $25-\mathrm{mg} \mathrm{mL}^{-1}$ dose of puromycin resulted in increased clearance of injected $70 \mathrm{kDa}$ dextran (Fig. 2A). Neither 1 nor $2 \mathrm{mg} \mathrm{mL}^{-1}$ adriamycin resulted in any increased clearance of $70 \mathrm{kDa}$ dextran up to $96 \mathrm{hpi}$ (Fig. 2B). The first derivative of puromycin-induced dextran clearance from the dorsal aorta shows that in all groups, the rate of change is highest over the initial $24 \mathrm{~h}$ postinjection (Fig. 2C). This is expected to be due to the continuing maturation of podocytes and the GFB structure between 3 and 4 dpf. $^{22,23,26,32}$ Over the first $24 \mathrm{~h}$, the $25-\mathrm{mg} \mathrm{mL}^{-1}$ dose of puromycin significantly increased the rate of loss of injected dextran compared with controls (Fig. 2C). The effect of increased dextran excretion by $25 \mathrm{mg} \mathrm{mL}^{-1}$ puromycin was also observed in fish injected at 4 dpf (Fig. 2D).

Despite having no effect on dextran clearance, the highest tested dose of adriamycin $\left(2 \mathrm{mg} \mathrm{mL}^{-1}\right)$ significantly decreased the heart rate (Fig. 2E) and blood flow (Fig. 2F). The highest dose of puromycin $\left(25 \mathrm{mg} \mathrm{mL}^{-1}\right)$ had no effect on heart rate, but reduced venous blood flow (Fig. 2F). Due to the lack of effect of adriamycin on dextran clearance and its side effects on heart rate and blood flow, this compound was not further assessed. Conversely, with its impact on dextran clearance, puromycin was further evaluated for its effects on podocyte ultrastructure.

\section{Analysis of puromycin-induced ultrastructural changes in the GFB}

To assess podocyte structure after $25 \mathrm{mg} \mathrm{mL}^{-1}$ puromycin injection, the ultrastructure of the GFB was quantitatively analyzed as performed by Benchimol et al. (Fig. 3A). ${ }^{44}$ Well- developed primary and secondary foot processes, SDs, and a trilaminar GBM were clearly visible at $4 \mathrm{dpf}$ in healthy zebrafish glomeruli (Fig. 3B, C). Focal podocyte effacement, the loss of filtration slits, and enlargement of the foot process area in contact with the GFB were observed as a result of $25 \mathrm{mg} \mathrm{mL}^{-1}$ puromycin (Fig. 3D, E). To estimate the time point at which podocyte effacement occurs, samples were taken at 8 and $24 \mathrm{hpi}$. Foot process frequency (Fig. 3F) and foot process width (Fig. 3G) were not significantly altered until $24 \mathrm{hpi}$, suggesting that significant puromycin-induced podocyte effacement likely occurs between 8 and $24 \mathrm{hpi}$.

\section{Measurement of dextran excretion dynamics using a modified 96-well plate assay}

Our validated puromycin injury model was used to test a novel assay we developed to minimize fish handling and to allow the dynamics of glomerular permeability to be measured in a semihigh-throughput manner. This method allows up to 48 fish to be studied simultaneously (Fig. 4A, B). The loss of glomerular selectivity by $25 \mathrm{mg} \mathrm{mL}^{-1}$ puromycin was also confirmed by our excretion assay (Fig. 4C). As similarly observed with the assay of FITC-dextran clearance from the dorsal aorta, the mean first derivative of the excretion assay shows that changes in dextran clearance are highest within $24 \mathrm{~h}$ of puromycin injection (Fig. 4D). This suggests that functional changes in the podocytes begin within $24 \mathrm{~h}$ postinjection.

\section{Discussion}

The utility of acute pharmacological glomerular injury models would be greatly increased if they induced podocyte regeneration and the subsequent dynamics of GFB repair 



FIG. 2. Comparison of adriamycin and puromycin as agents for podocyte injury. (A) Mean ( \pm SEM) dextran clearance is significantly increased by injection of $25 \mathrm{mg} \mathrm{mL}^{-1}$, but not by $5 \mathrm{mg} \mathrm{mL}^{-1}$, puromycin at $3 \mathrm{dpf}(n=8)$. (B) Neither $1 \mathrm{nor} 2 \mathrm{mg}$ $\mathrm{mL}^{-1}$ adriamycin injected at $3 \mathrm{dpf}$ has a significant effect on mean $( \pm$ SEM) dextran clearance $(n=8)$. (C) First derivative of (A) showing the rate change in dextran clearance is greatest within the first 24 hpi with the first derivative of $25 \mathrm{mg} \mathrm{mL}^{-1}$ puromycin being significantly greater than both controls and $5 \mathrm{mg} \mathrm{mL}^{-1}$-treated fish. (D) $25 \mathrm{mg} \mathrm{mL}^{-1}$ puromycin injected at $4 \mathrm{dpf}$ also significantly decreases mean $( \pm \mathrm{SEM})$ dextran clearance 24 hpi. (E) $2 \mathrm{mg} \mathrm{mL}^{-1}$ adriamycin significantly reduces mean $( \pm$ SEM) heart rate, and puromycin has no effect $(n=8)$. (F) Mean $( \pm$ SEM) venous blood flow is decreased by both $2 \mathrm{mg} \mathrm{mL}^{-1}$ adriamycin and $25 \mathrm{mg} \mathrm{mL}^{-1}$ puromycin $(n=8)$. Two-way ANOVA, $p$-value summary: $*<0.05$; $* * \leq 0.002$; $* * * \leq 0.0002$.

could be monitored. Unlike in human disease and experimental rodent models, zebrafish podocytes retain their regenerative capacity throughout their entire lifecycle. ${ }^{35-37}$ Current models of acute pharmacological podocyte injury in larval fish are limited by terminal edema. Edema is likely caused by a reduced renal flow, which impairs ion homeostasis due to a disruption in tubular ion uptake. ${ }^{39,46,48}$

To further the development of pharmacological glomerular injury methods in larval zebrafish, specific and off-target effects of puromycin and adriamycin were compared to identify a suitable compound for the induction and resolution of glomerular injury. To discriminate against effects arising from impaired podocyte development, podocyte toxins were administrated at $3 \mathrm{dpf}$. At this age, $70 \%$ the GBM is covered with organized podocyte foot processes and SDs, and the
GFB is capable of selective filtraiton. ${ }^{22,26,39}$ To track the dynamics of glomerular function, we developed a novel assay to monitor the onset and resolution of abnormal protein filtration arising from podocyte injury and repair.

Rodent models of adriamycin nephropathy are well established and characterized by increased protein excretion, tubulointerstitial inflammation, and fibrosis. ${ }^{15,49}$ In larval fish, waterborne adriamycin administered from 9 to $48 \mathrm{~h}$ postfertilization (hpf) impairs the establishment of discriminative filtration due to abnormal podocyte development. ${ }^{46}$ Waterborne delivery of adriamycin is also associated with off-target toxicity, particularly to the heart. ${ }^{15,46,50} \mathrm{We}$ aimed to reduce off-target effects by using a more direct delivery of adriamycin by intravenous microinjection, as similarly used in the rodent adriamycin nephropathy model. ${ }^{15}$ In our model 

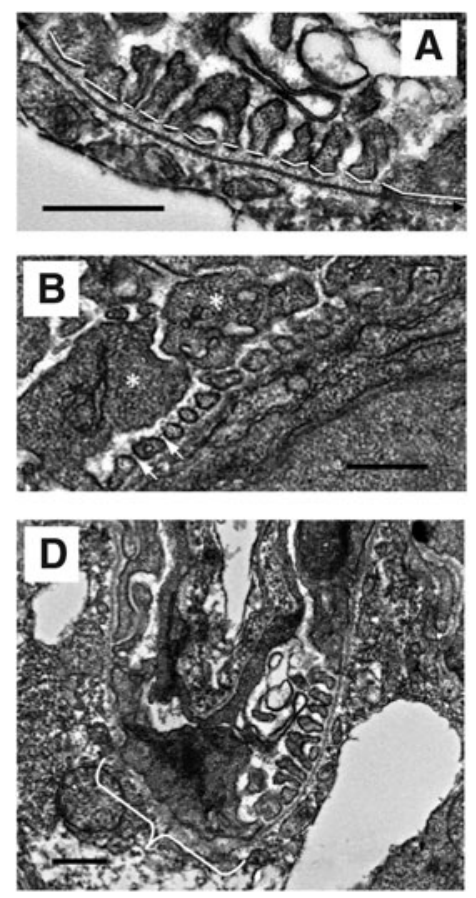

$\mathbf{F}$

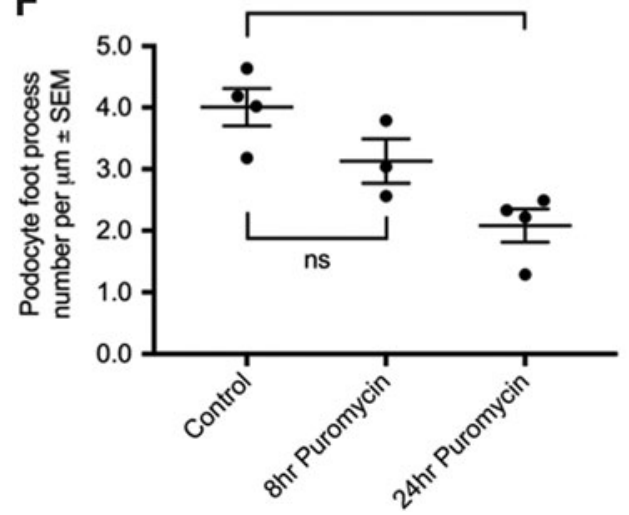

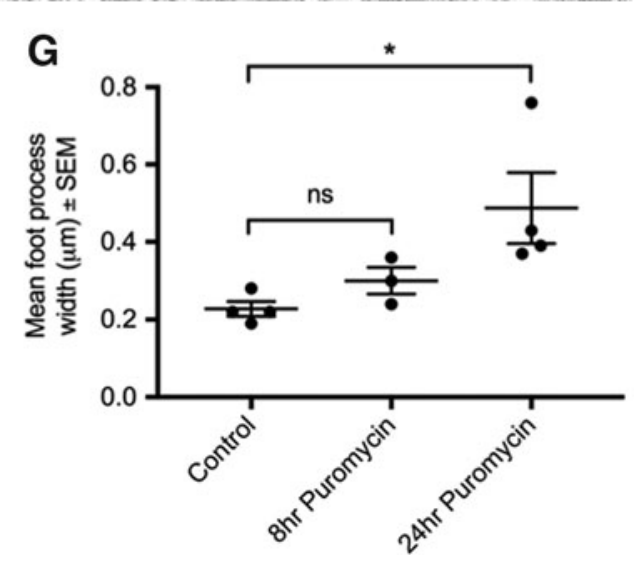
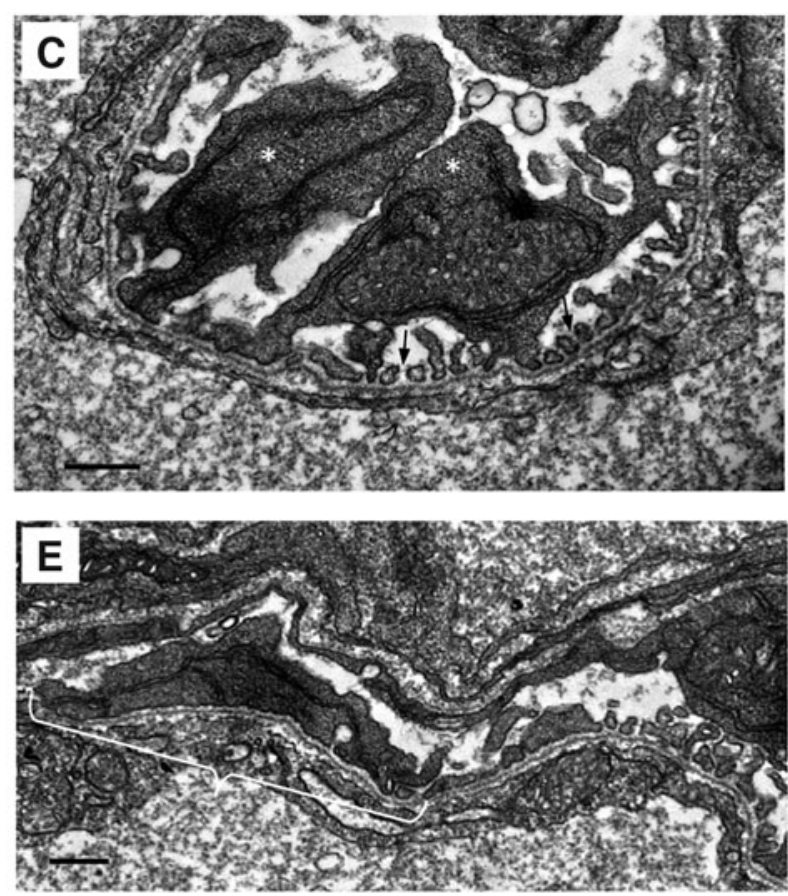

FIG. 3. Effect of intravenous puromycin injection at $3 \mathrm{dpf}$ on GFB structure $(n=3-4)$. (A) Image analysis of GFB for determination of podocyte effacement. Black double line $=$ total length of GBM. White lines $=$ foot process width. $(\mathbf{B}$, C) Representative transmission electron microscopy micrographs at $24 \mathrm{hpi}$ showing normal GFB structure with primary $(*)$ and secondary foot processes with slit diaphragms (arrows). Foot process morphology is not significantly different from that of sham fish at $8 \mathrm{hpi}$ (data not shown). (D, E) $25 \mathrm{mg} \mathrm{mL}^{-1}$ puromycin results in patchy podocyte effacement (white brackets). (F) Administration of $25 \mathrm{mg} \mathrm{mL}^{-1}$ puromycin significantly decreases the mean $( \pm$ SEM $)$ number of foot processes per unit length of GBM ( $p=$ $0.005)$ and $(\mathbf{G})$ mean $( \pm$ SEM $)$ foot process width $(p=0.04)$. Analysis by one-way ANOVA with post hoc Dunnet's test. Scale bars are all $0.5 \mu \mathrm{m}$. GFB, glomerular filtration barrier; GBM, glomerular basement membrane. $p$-value summary: $*<0.05 ; * * \leq 0.002$. assessing the effects of injected adriamycin in older larval fish with a functional glomerulus, the clearance of coinjected $70 \mathrm{kDa}$ dextran was likely confounded by off-target cardiovascular effects.

The pronephric glomerular arterioles of zebrafish branch directly off the dorsal aorta, thus glomerular flow is tightly associated with flow in the central vasculature. ${ }^{29,40}$ In the present study, the adriamycin-induced reduction in heart rate and blood flow may have decreased glomerular flow rates and masked any increase in dextran clearance resulting from podocyte damage. Due to confounding off-target cardiovascular effects, adriamycin was considered unsuitable for modeling glomerular injury in zebrafish older than $3 \mathrm{dpf}$.

The injection of puromycin at 3 or $4 \mathrm{dpf}$ in zebrafish larvae has been shown to result in podocyte effacement, loss of glomerular selectivity, and terminal edema. ${ }^{39,51}$ To mitigate off-target effects and edema, a lower dose of puromycin $(5 \mathrm{mg}$ $\mathrm{mL}^{-1}$ ) was tested, but was found insufficient for the measurable induction of glomerular injury. By injecting $1 \mathrm{~nL}$ of $25 \mathrm{mg}$ $\mathrm{mL}^{-1}$ puromycin, as opposed to the 2.3-4.6-nL volume used by used Hentschel et al., ${ }^{39}$ we observed similar effects on dextran clearance and podocyte effacement, including the onset of terminal edema. Injection volumes were restricted to $1 \mathrm{~nL}$ for the avoidance of negative effects on cardiovascular function, which can arise when injecting larger volumes into the circulation of larval fish (unpublished).

We employed image analysis ${ }^{44}$ to quantitate podocyte foot process width and foot process frequency. The quantification of podocyte morphology established that measurable structural changes occur between 8 and 24 hpi.

At a dose causing disruption of the GFB, puromycin also reduced blood flow by $24 \mathrm{hpi}$. Although decreased blood flow was observed, a higher dextran clearance was still detected, indicating a robust effect of puromycin on glomerular permeability. Previous studies have shown that FITC-dextran intensity in the vasculature is not significantly affected by edema-induced volume expansion. ${ }^{39}$ The dose of puromycin established for acute podocyte injury was not associated with any measurable effects on tubular structure (data not shown), including the brush border. The cilia of tubular cells that form the zebrafish nephron are essential for fluid clearance. ${ }^{52}$ This suggests that in the pronephros, effects of puromycin are specific to the glomerulus, as similarly reported by Hentschel et al. $^{39}$ 
A


B

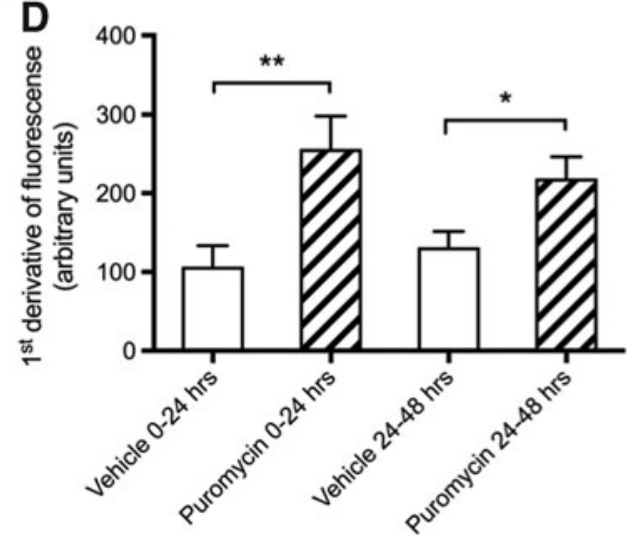

FIG. 4. Plate assay to measure dynamics of cleared $70 \mathrm{kDa}$ FITC-dextran following puromycin injection at $3 \mathrm{dpf}(n=14)$. (A) Photo of modified 96-well plate with 48 paired wells. White arrow pointing toward $250-\mu \mathrm{m}$ slits between paired wells. (B) Schematic of well pairing. A single fish injected with FITC-dextran is placed in well 1. FITC fluorescence from excreted dextran is assayed in well 2. (C) Twenty-five milligrams per milliliter puromycin significantly increases mean $( \pm$ SEM) clearance of $70 \mathrm{kDa}$ FITC-dextran over $48 \mathrm{hpi}$. Analysis of linear regression slopes shows that both are significantly different $(p=<0.0001)$. (D) The mean $( \pm$ SEM) first derivative of puromycin-treated fish between 0 and 24 hpi is $149 \pm 49.4$ higher than vehicle-treated fish. This is maintained, although to a lesser extent, over 24-48 hpi where the mean first derivative of puromycin-treated fish is $87.4 \pm 34.2$ higher than control fish. T-test, $p$-value: $*<0.02 ; * *<0.006$.

To advance methods for the in vivo analysis of glomerular permeability in zebrafish, we developed a novel assay of dextran excretion allowing the continuous monitoring of glomerular permeability to high-molecular-weight (HMW) molecules. Repeated handling and anesthesia of fish induce stress responses and cause detrimental effects on cardiovascular function. ${ }^{41,53,54}$ This limits the frequency and precision at which FITC-dextran clearance may be measured from the vasculature of larval fish. Hence, an advantage of our semihigh-throughput plate assay is that after injection of FITC-dextran, it involves no further handling or anesthesia of fish. This dextran excretion assay complemented measurements of FITC-dextran clearance from the dorsal aorta and confirmed that increased excretion of HMW molecules occurred in the first $24 \mathrm{~h}$ after injection of puromycin. While our dextran assays suggest that in some individuals glomerular function may resolve by $48 \mathrm{hpi}$, systemic effects leading to terminal edema were not resolved, as reported in previous studies. $^{39}$

Puromycin- or adriamycin-induced nephrosis are among the oldest and most widely used models of human nephrotic syndrome, particularly using rats due to their susceptibility to both compounds. ${ }^{55}$ This study has identified the suitability of puromycin for induction of acute podocyte injury in zebrafish larvae. As observed in rodent models, the defined single dose of puromycin used in the present study results in podocyte effacement with a concurrent loss of glomerular selectivity. The onset of GFB changes within $24 \mathrm{~h}$ in fish is considerably faster than occurs in rodent models, which have a 4-5-day latency period after puromycin injection. ${ }^{12,13,56}$

In contrast to rodent models, puromycin administration in larval fish results in off-target effects on central blood flow, which may result in the terminal onset of edema. ${ }^{39}$ Further studies are required to assess the correlation between edema and GFB changes to establish if puromycin-induced injury is recoverable in larval fish, as observed in the genetic podocyte NTR-based podocyte injury model. ${ }^{35,37} \mathrm{~A}$ pharmacological glomerular injury model is yet to be established in adult zebrafish. Unlike larval fish, which can only repair their single paired nephron, glomerular injury in adult fish may induce neonephrogenesis, as documented with tubular injury. ${ }^{36,57}$

Our novel excretion assay for the monitoring of glomerular selectivity, a proxy of podocyte function, enables a semihighthroughput and dynamic measurement of glomerular filtration without the confounding effects of repeated fish handling. This will facilitate the screening of small molecules that prevent glomerular injury or promote podocyte repair. The ability to measure the dynamics of GFB function provides an excellent tool for the in vivo analysis of glomerular injury and podocyte repair in larval zebrafish. 


\section{Acknowledgments}

This work was financially supported by Kidney Research United Kingdom and a British Heart Foundation Centre of Research Excellence award. The authors also acknowledge support from the Wellcome Trust for the zebrafish facility and acquisition of the electron microscope (Multiuser Equipment Grant, WT104915MA). The authors owe their gratitude to Stephen Mitchell for his expertise and processing of TEM samples.

\section{Disclosure Statement}

No competing financial interests exist.

\section{References}

1. Bertram JF, Douglas-Denton RN, Diouf B, Hughson MD, Hoy WE. Human nephron number: Implications for health and disease. Pediatri Nephrol (Berlin, Germany) 2011;26: 1529-1533.

2. Miner JH. The glomerular basement membrane. Exp Cell Res 2012;318:973-978.

3. Perico L, Conti S, Benigni A, Remuzzi G. Podocyte-actin dynamics in health and disease. Nat Rev Nephrol 2016;12: 692-710.

4. Grahammer F, Schell C, Huber TB. The podocyte slit diaphragm - from a thin grey line to a complex signalling hub. Nat Rev Nephrol 2013;9:587-598.

5. Kestila M, Lenkkeri U, Mannikko M, Lamerdin J, McCready P, Putaala $\mathrm{H}$, et al. Positionally cloned gene for a novel glomerular protein-nephrin-is mutated in congenital nephrotic syndrome. Mol Cell 1998;1:575-582.

6. Winn MP, Conlon PJ, Lynn KL, Farrington MK, Creazzo $\mathrm{T}$, Hawkins AF, et al. A mutation in the TRPC6 cation channel causes familial focal segmental glomerulosclerosis. Science 2005;308:1801-1804.

7. Mucha B, Ozaltin F, Hinkes BG, Hasselbacher K, Ruf RG, Schultheiss M, et al. Mutations in the Wilms' tumor 1 gene cause isolated steroid resistant nephrotic syndrome and occur in exons 8 and 9. Pediatr Res 2006;59:325-331.

8. Boute N, Gribouval O, Roselli S, Benessy F, Lee H, Fuchshuber A, et al. NPHS2, encoding the glomerular protein podocin, is mutated in autosomal recessive steroidresistant nephrotic syndrome. Nat Genet 2000;24:349-354.

9. Shankland SJ. The podocyte's response to injury: Role in proteinuria and glomerulosclerosis. Kidney Int 2006;69: 2131-2147.

10. Drumond MC, Kristal B, Myers BD, Deen WM. Structural basis for reduced glomerular filtration capacity in nephrotic humans. J Clin Invest 1994;94:1187-1195.

11. Siddall EC, Radhakrishnan J. The pathophysiology of edema formation in the nephrotic syndrome. Kidney Int 2012;82:635-642.

12. Zheng CX, Chen ZH, Zeng CH, Qin WS, Li LS, Liu ZH. Triptolide protects podocytes from puromycin aminonucleoside induced injury in vivo and in vitro. Kidney Int 2008;74:596-612.

13. Ryan GB, Karnovsky MJ. An ultrastructural study of the mechanisms of proteinuria in aminonucleoside nephrosis. Kidney Int 1975;8:219-232.

14. Kiffel J, Rahimzada Y, Trachtman H. Focal segmental glomerulosclerosis and chronic kidney disease in pediatric patients. Adv Chronic Kidney Dis 2011;18:332-338.
15. Lee VW, Harris DC. Adriamycin nephropathy: A model of focal segmental glomerulosclerosis. Nephrology (Carlton) 2011;16:30-38.

16. Westhoff JH, Giselbrecht S, Schmidts M, Schindler S, Beales PL, Tonshoff B, et al. Development of an automated imaging pipeline for the analysis of the zebrafish larval kidney. PLoS One 2013;8:e82137.

17. MacRae CA, Peterson RT. Zebrafish as tools for drug discovery. Nat Rev Drug Discov. 2015;14:721-731.

18. Wang G, Rajpurohit SK, Delaspre F, Walker SL, White DT, Ceasrine A, et al. First quantitative high-throughput screen in zebrafish identifies novel pathways for increasing pancreatic beta-cell mass. eLife 2015;4:1-26.

19. Patton EE, Zon LI. The art and design of genetic screens: Zebrafish. Nat Rev Genet 2001;2:956-966.

20. Noonan HR, Metelo AM, Kamei CN, Peterson RT, Drummond IA, Iliopoulos O. Loss of vhl in the zebrafish pronephros recapitulates early stages of human clear cell renal cell carcinoma. Dis Model Mech 2016;9:873-884.

21. Hanke N, Staggs L, Schroder P, Litteral J, Fleig S, Kaufeld J, et al. "Zebrafishing" for novel genes relevant to the glomerular filtration barrier. Biomed Res Int 2013;2013: 658270 .

22. Kramer-Zucker AG, Wiessner S, Jensen AM, Drummond IA. Organization of the pronephric filtration apparatus in zebrafish requires Nephrin, Podocin and the FERM domain protein Mosaic eyes. Dev Biol 2005;285:316-329.

23. Drummond IA, Majumdar A, Hentschel H, Elger M, Solnica-Krezel L, Schier AF, et al. Early development of the zebrafish pronephros and analysis of mutations affecting pronephric function. Development 1998;125:46554667.

24. Siegerist F, Blumenthal A, Zhou W, Endlich K, Endlich N. Acute podocyte injury is not a stimulus for podocytes to migrate along the glomerular basement membrane in zebrafish larvae. Sci Rep 2017;7:43655.

25. Siegerist F, Zhou W, Endlich K, Endlich N. 4D in vivo imaging of glomerular barrier function in a zebrafish podocyte injury model. Acta Physiol (Oxf) 2017;220:167173.

26. Zhu X, Chen Z, Zeng C, Wang L, Xu F, Hou Q, et al. Ultrastructural characterization of the pronephric glomerulus development in zebrafish. J Morphol 2016;277:11041112.

27. Drummond I. Making a zebrafish kidney: a tale of two tubes. Trends Cell Biol 2003;13:357-365.

28. Drummond IA.: The zebrafish pronephros: A genetic system for studies of kidney development. Pediatr Nephrol 2000;14:428-435.

29. Rider SA, Mullins LJ, Verdon RF, MacRae CA, Mullins JJ. Renin expression in developing zebrafish is associated with angiogenesis and requires the Notch pathway and endothelium. Am J Physiol Renal Physiol 2015;309:F531-F539.

30. Wingert RA, Davidson AJ. The zebrafish pronephros: A model to study nephron segmentation. Kidney Int 2008;73: 1120-1127.

31. Wingert RA, Selleck R, Yu J, Song HD, Chen Z, Song A, et al. The cdx genes and retinoic acid control the positioning and segmentation of the zebrafish pronephros. PLoS Genet 2007;3:1922-1938.

32. Ichimura K, Bubenshchikova E, Powell R, Fukuyo Y, Nakamura T, Tran U, et al. A comparative analysis of glomerulus development in the pronephros of Medaka and zebrafish. PLoS One 2012;7:e45286. 
33. Kroeger PT, Wingert RA. Using zebrafish to study podocyte genesis during kidney development and regeneration. Genesis 2014;52:771-792.

34. Cirio MC, de Caestecker MP, Hukriede NA. Zebrafish models of kidney damage and repair. Curr Pathobiol Rep 2015;3:163-170.

35. Huang J, McKee M, Huang HD, Xiang A, Davidson AJ, Lu HA. A zebrafish model of conditional targeted podocyte ablation and regeneration. Kidney Int 2013;83:1193-1200.

36. Zhou W, Boucher RC, Bollig F, Englert C, Hildebrandt F. Characterization of mesonephric development and regeneration using transgenic zebrafish. Am J Physiol Renal Physiol 2010;299:F1040-F1047.

37. Zhou W, Hildebrandt F. Inducible podocyte injury and proteinuria in transgenic zebrafish. J Am Soc Nephrol 2012;23:1039-1047.

38. Curado S, Stainier DY, Anderson RM. Nitroreductasemediated cell/tissue ablation in zebrafish: A spatially and temporally controlled ablation method with applications in developmental and regeneration studies. Nat Protoc 2008;3: 948-954.

39. Hentschel DM, Mengel M, Boehme L, Liebsch F, Albertin C, Bonventre JV, et al. Rapid screening of glomerular slit diaphragm integrity in larval zebrafish. Am J Physiol Renal Physiol 2007;293:F1746-F1750.

40. Rider SA, Tucker CS, del-Pozo J, Rose KN, MacRae CA, Bailey MA, et al. Techniques for the in vivo assessment of cardio-renal function in zebrafish (Danio rerio) larvae. J Physiol 2012;590:1803-1809.

41. Denvir MA, Tucker CS, Mullins JJ. Systolic and diastolic ventricular function in zebrafish embryos: influence of norepenephrine, MS-222 and temperature. BMC Biotechnol 2008;8:21.

42. Isogai S, Horiguchi M, Weinstein BM. The vascular anatomy of the developing zebrafish: An atlas of embryonic and early larval development. Dev Biol 2001;230:278-301.

43. Lyons DA, Naylor SG, Mercurio S, Dominguez C, Talbot WS. KBP is essential for axonal structure, outgrowth and maintenance in zebrafish, providing insight into the cellular basis of Goldberg-Shprintzen syndrome. Development 2008; 135:599-608.

44. Benchimol de Souza D, Gregório BM, Benchimol M, Nascimento FM. Evaluation of the glomerular filtration barrier by electron microscopy. In: Modern Electron Microscopy in Physical and Life Sciences. Janecek M and Kral R (eds), pp. 187-206, InTech, Rijeka, Croatia, 2016.

45. Yin W, Zhang J, Jiang Y, Juan S. Combination therapy with low molecular weight heparin and Adriamycin results in decreased breast cancer cell metastasis in $\mathrm{C}(3) \mathrm{H}$ mice. Exp Ther Med 2014;8:1213-1218.

46. Zennaro C, Mariotti M, Carraro M, Pasqualetti S, Corbelli A, Armelloni S, et al. Podocyte developmental defects caused by adriamycin in zebrafish embryos and larvae:
A novel model of glomerular damage. PLoS One 2014;9: e98131.

47. He B, Ebarasi L, Hultenby K, Tryggvason K, Betsholtz C. Podocin-green fluorescence protein allows visualization and functional analysis of podocytes. J Am Soc Nephrol 2011;22:1019-1023.

48. McKee RA, Wingert RA. Zebrafish renal pathology: Emerging models of acute kidney injury. Curr Pathobiol Rep 2015;3:171-181.

49. Rocke J, Lees J, Packham I, Chico T. The zebrafish as a novel tool for cardiovascular drug discovery. Recent Pat Cardiovasc Drug Discov 2009;4:1-5.

50. Liu Y, Asnani A, Zou L, Bentley VL, Yu M, Wang Y, et al. Visnagin protects against doxorubicin-induced cardiomyopathy through modulation of mitochondrial malate dehydrogenase. Sci Transl Med 2014;6:266ra170.

51. Burford JL, Gyarmati G, Shirato I, Kriz W, Lemley KV, Peti-Peterdi J. Combined use of electron microscopy and intravital imaging captures morphological and functional features of podocyte detachment. Pflugers Arch 2017;469: 965-974.

52. Kramer-Zucker AG, Olale F, Haycraft CJ, Yoder BK, Schier AF, Drummond IA. Cilia-driven fluid flow in the zebrafish pronephros, brain and Kupffer's vesicle is required for normal organogenesis. Development 2005;132: 1907-1921.

53. Carter KM, Woodley CM, Brown RS. A review of tricaine methanesulfonate for anesthesia of fish. Rev Fish Biol Fish 2011;21:51-59.

54. Beckman M. Therapeutic review: Tricaine Methanesulfonate. J Exotic Pet Med 2016;25:261-263.

55. Smeets B, Moeller MJ. Models of FSGS and minimal change nephropathy. Drug Disc Today Dis Models 2010;7: 3-11.

56. Hagiwara M, Yamagata K, Capaldi RA, Koyama A. Mitochondrial dysfunction in focal segmental glomerulosclerosis of puromycin aminonucleoside nephrosis. Kidney Int 2006;69:1146-1152.

57. McCampbell KK, Springer KN, Wingert RA. Atlas of Cellular Dynamics during Zebrafish Adult Kidney Regeneration. Stem Cells Int 2015;2015:547636.

Address correspondence to:

Sebastien Andrew Rider, PhD, MSc, BSc (hons)

The University/BHF Centre for Cardiovascular Science

The University of Edinburgh

Queens Medical Research Institute

47 Little France Crescent

Edinburgh EH16 4TJ United Kingdom

E-mail: srider@staffmail.ed.ac.uk 